\title{
Out of Field Breast Doses as an Undesired Consequence of Cervical Cancer Radiotherapy
}

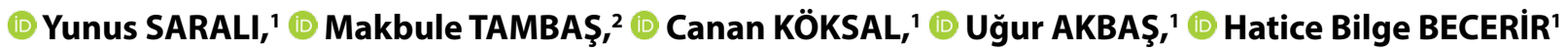 \\ 'Department of Medical Physics, İstanbul University, Çapa, Institute of Oncology, İstanbul-Turkey \\ ${ }^{2}$ Department of Radiation Oncology, Okmeydanı Training and Research Hospital, İstanbul-Turkey
}

\begin{abstract}
OBJECTIVE
Unwanted doses may occur in distant organs, outside of the region where we want to be irradiated in patients treated with radiotherapy. These doses cannot be accounted accurately by the treatment planning system (TPS) yet. In our study, the doses received by the breast tissue outside the field of irradiation are aimed to investigate dosimetrically in irradiation with three-dimensional conformal radiotherapy (3DCRT), intensity-modulated radiation therapy (IMRT) and volumetric arc therapy (VMAT) techniques for cervical cancer.
\end{abstract}

\section{METHODS}

The patient was simulated in Alderson Rando phantom and irradiated with three different techniques in Varian DHX (Rapidarc) linear accelerator with 48 Gy in 24 fractions. Doses that occur in the breast tissue were measured using thermoluminescent dosimeters and compared with each other and the data obtained from TPS.

\section{RESULTS}

The dose values in the right and left breasts were found to be statistically similar to each other ( $>0.05)$, whereas significant differences were detected between different techniques. The mean calculated breast doses were 7.16 $\pm 1.61 \mathrm{cGy}$ in 3D-CRT, 27,75 $\pm 3,88 \mathrm{cGy}$ in IMRT and 12,20 $\pm 2,65 \mathrm{cGy}$ in VMAT, respectively.

\section{CONCLUSION}

The breast tissue doses are significantly lower in 3D-CRT and VMAT compared with IMRT. This finding should be considered while choosing a treatment technique, especially in young patients with cervical cancer.

Keywords: Breast dose; cervical cancer radiotherapy; out-of-field dose; peripheral dose.

Copyright $\odot$ 2020, Turkish Society for Radiation Oncology

\section{Introduction}

The incidence of cervical cancer was 528.000 in 2012, while 266.000 patients died due to cervical cancer during the same year.[1] Although it is the fourth common cancer in women globally, cervical cancer is the main cause of death among women cancer patients in developing countries where $85 \%$ of cases are diagnosed.[1-3]

Radiotherapy is the main component of cervical cancer treatment in both primary and adjuvant setting. The dose to the radiation-sensitive breast tissue located 
outside the treatment field is important, particularly in younger cervical cancer patients who will receive radiotherapy. Healthy cells in the surrounding tissue can be damaged while tumor cells are irradiated during radiotherapy. The dose-dependent biological effects of radiation may arise as early or late side effects. To decrease these side effects, the maximum level of protection for normal tissues should be provided.[4]

Despite achieving higher conformality with modern techniques, unwanted doses may occur in the field outside the region to be irradiated. These doses are called out-of-field doses (peripheral doses, PD). Due to the stochastic effects of ionizing radiation and the risk of secondary cancers, PD should be determined by measuring them. They emerge due to the treatment head of medical linear accelerator, the collimator device, the scattering from the patient's body and the leakage.[5]

The potential risk of secondary cancers due to low doses of ionizing radiation is the main problem. The advanced treatment techniques, such as threedimensional conformal radiotherapy (3D-CRT) and intensity-modulated radiation therapy (IMRT), tend to increase radiation dose at organs out of the radiation treatment field. In addition, the risk of secondary cancer has become important since the average life expectancy of cancer patients has increased with developments in treatment techniques.

The very low doses received by organs distant to tumor volume during radiotherapy are known to cause secondary cancers. These doses cannot be calculated accurately by TPS.[6] Although breast tissue remains out of the radiation field in cervical cancer radiotherapy, doses that can be determined by measurements with phantom constitutes in breast tissues due to internal scattering and collimators. Therefore, measurement of PD using human-like phantoms is warranted.

In this study, the PD in radiation-sensitive breast tissue measured during irradiation of human-like Alderson Rando phantom for virtually created postoperative stage IB-II cervical cancer with 3D-CRT, IMRT and VMAT treatment technique and dose values calculated are compared.

\section{Materials and Methods}

\section{The Characteristics and Calibration of TLDs}

GR-200A thermoluminescent dosimeters (TLDs) are manufactured by Fimel company. TL rods are LiF originated dosimeters that are activated with $\mathrm{Mg}, \mathrm{Ca}$ and $\mathrm{P}$ and capable of measuring in the range of $0.5 \mu \mathrm{Gy}$ and
12 Gy with high sensitivity. The round TLD rods with a radius of $4.5 \mathrm{~mm}$ and a height of $0.8 \mathrm{~mm}$ were used in our study.

Standard deviation (SD) of the grouped TLD GR$200 \mathrm{~A}$ rods was less than \pm 1.5 was used in our study. Twelve TLD in the right breast and Twelve TLD in left breasts were used. Also, 4 TLDs were stored as a reserve. Two of 12 TLDs were selected for calibration in both breasts.

The response of the TLDs to radiation was determined before the measurements. TLDs were reset by annealing in the TLD oven. Firstly, TLD GR-200A round rods were passed through a baking process in the TLD oven at $220^{\circ} \mathrm{C}$ for 15 minutes. Then, 80 TLD GR-200A rods were irradiated with a source-skin distance (SSD) of $80 \mathrm{~cm}$, in a $20 \times 20 \mathrm{~cm}^{2}$ area at a depth of $5 \mathrm{~cm}$ to be exposed to $100 \mathrm{cGy}$ using specially designed PTW brand RW3 solid water phantom in Cirus Co-60 machine. To create a backscattering effect, a phantom of $10 \mathrm{~cm}$ thickness was placed under the rods. For calibration, TLDs were irradiated for $100 \mathrm{cGy}$ dose at a depth of $10 \mathrm{~cm}$ on the Rapidarc linear accelerator using 15 MV energy X-ray, which was used for three techniques during the planning phase. The absorbed dose values of each TLD were found by calculating the ratio between the values read during the measurements and the average of the responses of these calibration TLDs.

\section{The Characteristics of Phantom}

The phantom used in this study had no limbs and represented a $155 \mathrm{~cm}$ in length woman with breast tissue (Fig. 1). The breast tissue doses that occurred about 40 $\mathrm{cm}$ away from the center of the cervical tumor created virtually on the phantom were measured and evaluated. The original cross-sections of the phantom were used as the breast sections. The two parts of the upper sections of the breasts were removed. The TLDs placed in the breast sections are shown in Figure 2.

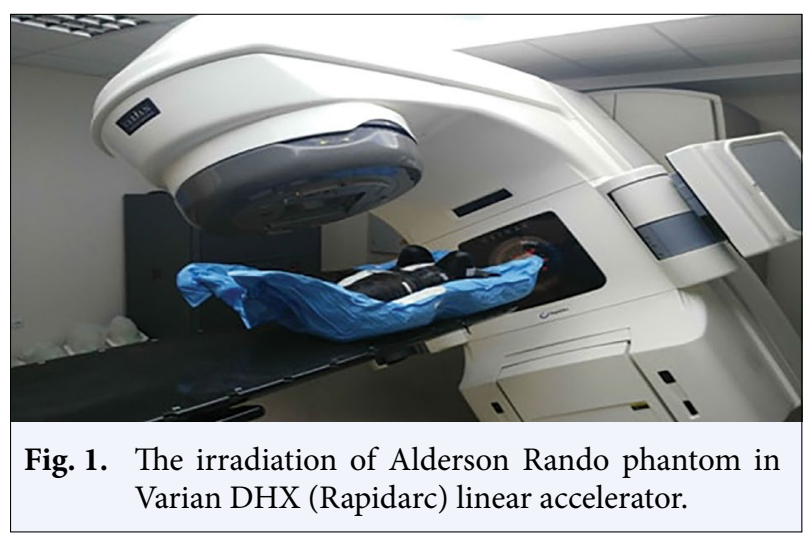




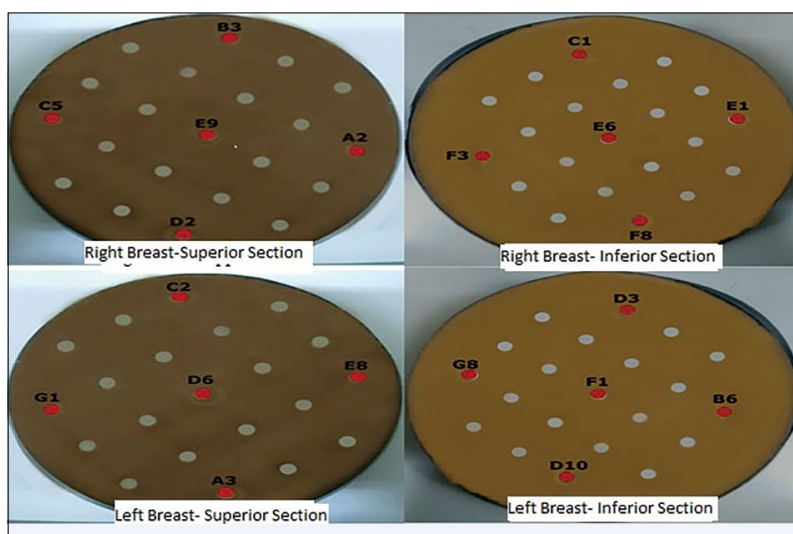

Fig. 2. The display of TLD rode sequences in the left and right breast sections used in the measurement.

\section{The Characteristics of Radiotherapy Plans}

The images of Alderson Rando phantom with $3 \mathrm{~mm}$ sections were taken in computed tomography and transferred to the Eclipse TPS. The right and left femoral heads, rectum, bladder, bowel volumes were delineated as critical organs. PTV was created with 1 $\mathrm{cm}$ of safety margin to CTV.

In the study, treatment plans simulating operated stage IB-II cervical cancer were prepared. The 3D-CRT, IMRT, and VMAT treatment plans were created using the AAA algorithm in Eclipse TPS. The center of PTV volume was chosen as the isocenter in all plans. Doses of virtual plans for all techniques were defined as 200 cGy daily with a total dose of 4800 cGy.

3D-CRT plans were created with four different beams with gantry angle $0^{\circ}, 90^{\circ}, 180^{\circ}, 270^{\circ}$ using $15-$ MV energy X-ray (box technique). To obtain a homogeneous dose distribution, $10^{\circ}$ virtual wedges were used in beams with $90^{\circ}$ and $270^{\circ}$ gantry angle.

Seven different beams with gantry angle $0^{\circ}, 52^{\circ}$, $104^{\circ}, 156^{\circ}, 208^{\circ}, 260^{\circ}, 312^{\circ}$ using $15-\mathrm{MV}$ energy X-ray were used to create IMRT plans. The collimator angle was set at $0 \mathrm{o}$ in each area. Sliding Window (SW) was selected as the irradiation technique in all of the IMRT fields. The optimization process was performed by defining dose values for PTV and the tolerance doses for critical organs, and the IMRT plan was created with dose calculation.

A double-arc plan was created using a $15-\mathrm{MV}$ energy X-ray for VMAT plan. Two full arcs by selecting the angle of $179.9^{\circ}-180.1^{\circ}$ and the collimator angle of $30^{\circ}$ in the first arc and the gantry angle of $180.1^{\circ}$ $-179.9^{\circ}$ and collimator angle of $330^{\circ}$ in the second arc beam were used. The dose rate that was selected to be a maximum of 600 monitor unit (MU)/min. varied dynamically according to the data entered optimization for during irradiation. VMAT plan was created by defining the desired dose values for PTV and the tolerance doses for critical organs.

Conformity index (CI) is defined as a ratio between planning target volume and treated volume (TV) at International Commission on Radiation Units and Measurements (ICRU) Report 62, CI=PTV/TV (equ 1). CI is ideal if this ratio is 1 . Homogeneity index (HI) was calculated according to the Formula at ICRU Report 83: $\mathrm{HI}=\left(\mathrm{D}_{\% 2}-\mathrm{D}_{\% 98}\right) / \mathrm{D}_{\% 50}$ (equ 2) where $\mathrm{D}_{\% 2}=$ The dose reached in $2 \%$ of the PTV volume, $\mathrm{D}_{0698}=$ The dose reached in $98 \%$ of the PTV volume and $\mathrm{D}_{\% 50}=$ The dose reached in $50 \%$ of the PTV volume. The dose distribution in PTV is highly homogeneous if $\mathrm{HI}$ approaches 0 . The CI and HI values for PTV-cervix and the organ at risk doses in plans with 3D-CRT, IMRT and VMAT techniques are compared.

The PD values measured and obtained from TPS were analyzed and compared with the Wilcoxon sign rank test using SPSS statistical software. Binary comparison of the out of field dose values in different treatment techniques were carried out as 3D-CRT vs. IMRT, 3D-CRT vs. VMAT, IMRT vs. VMAT using the Wilcoxon signed-rank test.

\section{Results}

PTV was intended to receive the prescribed dose, while critical organ doses were purposed to be below the acceptable limit doses while planning. The $\mathrm{CI}$ and $\mathrm{HI}$ values for PTV-cervix and the organ at risk doses in plans with 3D-CRT, IMRT and VMAT techniques are shown in Table 1 and 2.

Each of the plans was irradiated three times. The accumulated dose in the TLD rods of which arrangements on the breast tissue were indicated in Table 3 was calculated as the average dose value of three irradiations.

Table $1 \mathrm{Cl}$ and $\mathrm{HI}$ values for PTV-cervix in three techniques

\begin{tabular}{lccc} 
& 3D-CRT & IMRT & VMAT \\
\hline $\mathrm{D}_{\% 98}$ (cGy) & 4692 & 4781 & 4730 \\
$\mathrm{D}_{\% 2}$ (cGy) & 5147 & 5001 & 5006 \\
$\mathrm{D}_{\% 50}(\mathrm{cGy})$ & 4984 & 4943 & 4923 \\
TV (cc) & 767.5 & 782.5 & 764.5 \\
PTV (cc) & 804.2 & 804.2 & 804.2 \\
Cl & 0.954 & 0.973 & 0.951 \\
$\mathrm{HI}$ & 0.091 & 0.044 & 0.056 \\
\hline
\end{tabular}




\begin{tabular}{llccc} 
Table 2 & $\begin{array}{l}\text { The dose-volume histogram values for the } \\
\text { three techniques }\end{array}$ \\
& & $\begin{array}{c}\text { 3D-CRT } \\
\text { (cGy) }\end{array}$ & $\begin{array}{c}\text { IMRT } \\
\text { (cGy) }\end{array}$ & $\begin{array}{c}\text { VMAT } \\
\text { (cGy) }\end{array}$ \\
\hline PTV & & 4810 & 4827 & 4800 \\
Rectum & $\mathrm{D}_{\% 95}$ & 3426 & 2347 & 3050 \\
Bladder & $\mathrm{D}_{\% 60}$ & 4319 & 2966 & 3257 \\
& $\mathrm{D}_{\% 50}$ & 4469 & 3311 & 3920 \\
Bowel & $\mathrm{D}_{\% 35}$ & 3116 & 2402 & 3260 \\
Femoral heads & $\mathrm{D}_{\% 55}$ & 2715 & 2903 & 2588 \\
\multicolumn{1}{c}{ Right } & $\mathrm{D}_{\% 30}$ & & & \\
& $\mathrm{D}_{\% 15}$ & 1930 & 1295 & 1155 \\
\multicolumn{1}{c}{ Left } & $\mathrm{D}_{\% 55}$ & 2757 & 2466 & 2322 \\
& $\mathrm{D}_{\% 15}$ & 2103 & 1262 & 1053 \\
& $\mathrm{D}_{\% 5}$ & 2773 & 2206 & 1994 \\
\hline
\end{tabular}

The dose values in TLD rods placed in the upper, lower, right, left and the center point of the two sections of the right and left breast were measured and shown in Table 3. The average dose values of right and left breast were measured as 7,11 and 7,21 cGy in the 3D-CRT technique; 27,41 and $28,10 \mathrm{cGy}$ in the IMRT technique; 12,60 and $11,81 \mathrm{cGy}$ in VMAT technique, respectively. The dose differences between right and left breasts, as well as lower and upper sections, showed statistically similar results to each other ( $p>0.05)$. Since the difference between the two breasts dose was not significant, the averages of the measured dose with the TLD rods in both breasts for three techniques were calculated and compared with the dose values obtained from TPS and each other (Table 3). Also, the comparison of the average values of the accumulated doses in whole breast tissue was presented graphically in Figure 3.

The average breast doses were found as $7.16 \pm 1.61$ cGy in 3D-CRT, $27.75 \pm 3.88$ cGy in IMRT, and $12.20 \pm 2.65$ cGy in VMAT techniques, respectively (for 3D-CRT vs. IMRT, 3D-CRT vs. VMAT, IMRT vs. VMAT, $\mathrm{p}<0.01)$. On the other hand, breast dose values in TPS were zero, which indicated that the doses measured by TLDs could not be calculated by the TPS algorithm. In addition, MU values were 676, 1084 and $2228 \mathrm{MU}$ for 3D-CRT, VMAT, and IMRT, respectively. Taking into account the measured values out of field breast doses and MU values in three techniques, it can be clearly seen that breast doses increase in parallel with MU values (Table 4).

Since the treatment dose was prescribed as 4800 cGy in our planning, breast tissue received approximately $0.15 \%$ of the treatment dose in the 3D-CRT technique. The findings showed that the breast tissue

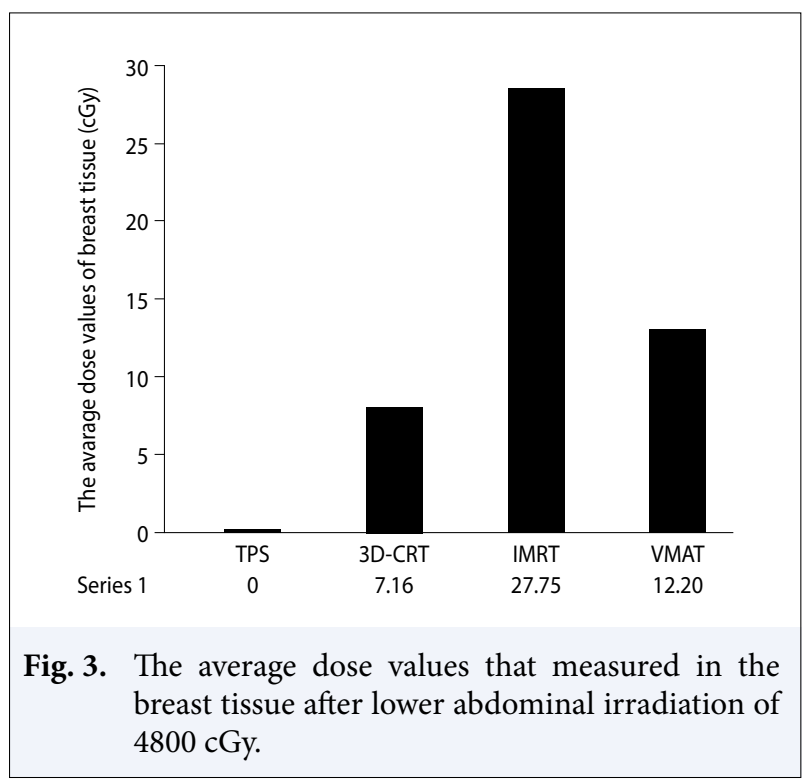

doses after irradiation were approximately $0.58 \%$ and $0.25 \%$ of the treatment dose in IMRT and VMAT techniques, respectively.

When three techniques were compared, the findings indicated that the IMRT method caused nearly four times more doses in breast tissue compared with the 3D-CRT method during lower abdominal irradiation of patient with cervical cancer. In addition, the IMRT technique caused twice more doses in breast tissue compared with VMAT technique, whereas the VMAT technique led to 1.5 times more peripheral doses than the 3D-CRT technique.

\section{Discussion}

The dose source outside the target volume is based on the following three basic parameters: 1 . The photon leakage from the head of the device, 2. The head structure of the device and the radiation scattered by the beam modifiers used, 3 . The scattered radiation generated by the treatment beam inside the patient. In addition to these are the neutrons which are generated at the head of the device in the $>10 \mathrm{MV}$ energy rays and photoneutrons created by these rays inside the patient. The contributions of the collimator scatter, head leakage and patient scatter to the PD have been studied by many researchers. In some of these studies, the findings showed that the contribution of leakage is larger, especially for areas near the edge of the field. PD reduces exponentially with distance from the area, whereas it also increases with the size of the radiation field.[7,8] In our study, the radiation treatment field, 
Table 3 The measured and calculated breast tissue out-of-field dose values for total prescribed dose in the three techniques and the TPS

\begin{tabular}{|c|c|c|c|c|c|}
\hline Location & TLD Name & $\begin{array}{c}\text { 3D-CRT } \\
\text { (cGy) }\end{array}$ & $\begin{array}{l}\text { IMRT } \\
\text { (cGy) }\end{array}$ & $\begin{array}{l}\text { VMAT } \\
\text { (cGy) }\end{array}$ & $\begin{array}{c}\text { TPS } \\
\text { (cGy) }\end{array}$ \\
\hline \multirow[t]{5}{*}{ Right breast inferior section } & C1-Superior & 5.31 & 23.80 & 9.56 & 0 \\
\hline & E1-Medial & 6.53 & 26.72 & 11.94 & 0 \\
\hline & E6-Center & 7.12 & 30.34 & 12.74 & 0 \\
\hline & F3-Lateral & 6.55 & 25.99 & 11.78 & 0 \\
\hline & F8-Inferior & 9.16 & 34.05 & 17.24 & 0 \\
\hline \multicolumn{6}{|l|}{ Right breast superior section } \\
\hline & B3-Superior & 4.93 & 22.49 & 9.07 & 0 \\
\hline & A2-Medial & 7.59 & 26.30 & 12.29 & 0 \\
\hline & E9-Center & 7.06 & 29.15 & 12.86 & 0 \\
\hline & C5-Lateral & 6.68 & 24.81 & 11.58 & 0 \\
\hline & D2-Inferior & 10.18 & 30.44 & 16.95 & 0 \\
\hline \multicolumn{6}{|l|}{ Left breast inferior section } \\
\hline & D3-Superior & 5.29 & 23.60 & 8.85 & 0 \\
\hline & B6-Lateral & 6.49 & 28.02 & 11.25 & 0 \\
\hline & F1-Center & 7.24 & 31.96 & 12.38 & 0 \\
\hline & G8-Medial & 6.89 & 27.46 & 11.10 & 0 \\
\hline & D10-Inferior & 9.87 & 34.90 & 16.41 & 0 \\
\hline \multicolumn{6}{|l|}{ Left breast superior section } \\
\hline & C2-Superior & 5.21 & 20.96 & 8.25 & 0 \\
\hline & E8-Lateral & 9.03 & 26.96 & 11.64 & 0 \\
\hline & D6-Center & 7.02 & 29.23 & 11.83 & 0 \\
\hline & G1-Medial & 5.61 & 24.82 & 10.17 & 0 \\
\hline & A3-Inferior & 9.47 & 33.08 & 16.19 & 0 \\
\hline Average doses & & 7.16 & 27.75 & 12.20 & 0 \\
\hline Standard deviation & & 1.61 & 3.88 & 2.65 & 0 \\
\hline & 3D-CRT vs. IMRT & 3D-CRT vs. VMAT & IMRT vs. VMAT & & \\
\hline Statistical difference & $p<0.01$ & $p<0.01$ & $p<0.01$ & & \\
\hline
\end{tabular}

TLD: Thermoluminescent dosimeter; 3D-CRT: Three-dimensional conformal radiotherapy; IMRT (cGy): Intensity-modulated radiation therapy; VMAT:Volumetric arc therapy; TPS: Treatment planning system

Table 4 The Monitor Unit (MU) and out of field breast doses in 3D-CRT, VMAT, and IMRT plans

\begin{tabular}{lcc} 
& $\begin{array}{c}\text { Monitor Units } \\
\text { (MU) }\end{array}$ & $\begin{array}{c}\text { Out of Field Breast } \\
\text { Doses (cGy) }\end{array}$ \\
\hline 3D-CRT & 676 & $7.16 \pm 1.61$ \\
VMAT & 1084 & $12.20 \pm 2.65$ \\
IMRT & 2228 & $27.75 \pm 3.88$ \\
\hline
\end{tabular}

3D-CRT: Three-dimensional conformal radiotherapy; VMAT: Volumetric arc therapy; IMRT: Intensity-modulated radiation therapy

PTV volume, and the distance between treatment volume and breast tissue were the same for all three techniques. The change in the radiotherapy techniques we used depended on the technique, the number of fields, the angles of the fields and the treatment times. The measured breast dose difference between the tech- niques can be explained by the difference in leakage and scatter from the device, in respect of the MU values of the plans. The measurements do not include the contribution of the neutrons.

To our knowledge, there is no one to one overlapping study with our study. However, several studies have been conducted on PD. Jia et al. investigated PD in irradiation for cervical cancer using X-rays with 6-MV energy (Elekta Synergy Linear Accelerator) with VMAT and step-and-shoot IMRT techniques. They measured the doses of the thyroid, breast tissue and lens using the ionization chamber while irradiating a human-like phantom formed by combining solid water phantom for a therapeutic dose of $5000 \mathrm{cGy}$. The total breast doses were found as 12.8 and $22.6 \mathrm{cGy}$ in VMAT and IMRT techniques, respectively.[8] Their results are consistent with our study. The small difference can be 
considered as the reasons for linear accelerator difference, used a different dosimetric method, energy used in treatment or size of the target volume.

Mansour et al. compared PD values between 3DCRT and IMRT techniques (Varian Ex Linear Accelerator) in pediatric radiotherapy. They observed lower PD values in the area near the target volume for the IMRT technique, which was explained by the potentially decreased amount of internal scattering due to irradiation with smaller domains in the sliding-window IMRT technique. In contrast, IMRT caused higher PD values in the areas distant to the treatment field compared with the 3D-CRT technique of which cause was suggested to be the higher MU values as and the leaks from linear accelerator treatment head. This is consistent with the results of our study since measured breast doses were found to be increased in parallel with MU values in three techniques. For both techniques, dose in nearby areas had a much smaller value compared with those in remote areas and the total of PD were close to each other.[9]

Cemile et al. prepared four treatment plans using $6 \mathrm{MV}$ X-ray energy, anteroposterior-posteroanterior (AP-PA), noncoplanar 3D-CRT, IMRT and noncoplanar IMRT treatment techniques for bilateral lung irradiation. They placed TLDs in RANDO phantom at five separate points outside the treatment area (brain, thyroid, left kidney, right kidney and umbilicus) and measured doses on the Siemens AvantGadre linear accelerator device. As a result of the measurements, they found that the dose amount was higher in the thyroid organ which was closer to the treatment area and the dose amount was lower in the umbilicus which was farther away from the treatment area for each technique.[10]

Cho et al. identified the risk of secondary cancer in the out-of-field regions for the neck, thorax and prostate regions 3D-CRT applications (Siemens Onko$\mathrm{r}^{\mathrm{tm}}$ Linear Accelerator). They found the dose of breast tissue as 0.7 and 0.9 cGy for a daily dose of $200 \mathrm{cGy}$ using the two energies, 6 and $10 \mathrm{MV}$ X-ray energy, for prostate plan measurements, respectively.[6]

Martín et al. investigated peripheral uterus dose in breast radiotherapy with plans consisting of two opposing tangential field with 6-MV X-ray energy with and without wedges (Saturne 40 Linear Accelerator). When a treatment dose of $5000 \mathrm{cGy}$ was applied, the doses of the uterus were measured as 4 and 4.2 cGy with and without wedges, respectively. In addition, the insufficiency of TPS in calculating PD was discussed in their study.[11] Similarly, Lee et al. measured bladder PD after breast cancer irradiation of $5040 \mathrm{cGy}$ with 3D-CRT, IMRT and VMAT (Varian Linear Accelera- tor) and found that accumulated bladder dose values were $2 \pm 0.1,9 \pm 0.4$ and $7 \pm 0.2 \mathrm{cGy}$, respectively.[12] These data also support the results of the present study.

The use of the protective set to protect the organs from the radiation scattered from collimator may result in up to twice reduced risk of secondary cancers development.[13] For instance, Banaee et al. reported that the use of a protective set for testes in male patients during irradiation with high energies (6 and 18 MV, Varian Linear Accelerator) for the tumors of the pelvic area led to $40-70 \%$ decrease in testes doses.[14] Similarly, the benefits of applying a protective set on breast tissue during cervical irradiation should be investigated in a further study.

Kase et al. measured the PD for open fields of different sizes and defined $\mathrm{PD}$ as the exponential function of distance for all the energies and field sizes (using G. E. Maxitron for $300 \mathrm{kVp}$, Varian Clinac 4 for $4 \mathrm{MV} \mathrm{X}$ rays, Siemens Mevatron XII for 8 MV X rays, Picker C-3000/75 for Co60 Gamma Rays). They found that PD depended on the collimators, field size and distance to the treatment field and the contribution of linear accelerator collimator to the PD dose had a value between $20 \%$ and $40 \%$. Although the contribution of radiation leaks to PD was less than other components, they determined that leaks became the dominant component at $60 \mathrm{~cm}$ and over distances from the central axis.[13]

Yousif et al. found the PD in distant organs by applying current calculation methods in cervical cancer patients irradiated with the AP-PA field in the Cobalt-60 device. For treatment doses in the range of $8400 \pm 600.1$ cGy to patients, they found average dose values of $62 \pm 9.6 \mathrm{cGy}, 56.9 \pm 7.5 \mathrm{cGy}, 93 \pm 12.3$ and $74.8 \pm 11.6 \mathrm{cGy}$ in the liver, spleen, right and left kidneys, respectively. [15] The results of this study support the PD data found in our study if the total treatment doses applied, the location of organs, and their distance from the radiation field are considered.

The measurement of PD is important in the assessment of secondary cancer risk potential. Kinhikar et al. compared the PD in the Varian Trilogy and HIart II tomotherapy (TT) devices with measurements using diode and TLDs in patients irradiated for head and neck cancer (HNC) with IMRT method. They detected that the TT device constituted 1.2-1.5 times less PD compared with the Trilogy device.[16] In addition, D’Agostino et al. compared PD in radiotherapy plans with IMRT, VMAT and TT technique (using Varian Clinac $2100 \mathrm{C} / \mathrm{D}$ Linear Accelerator for $10 \mathrm{MV}$ and 18 $\mathrm{MV}$, Tomotherapy for $6 \mathrm{MV}$ ) for prostate and HNC. The average PD per Gy in the thoracic region after 
prostate cancer irradiation with IMRT (18 MV), IMRT (10 MV) and TT (6 MV) methods were found as 3.25 mGy, $1.45 \mathrm{mGy}$ and $1.09 \mathrm{mGy}$, respectively. Furthermore, the average PD per Gy in the pelvic region after HNC irradiation with VMAT (6 MV) and TT (6MV) methods were measured as $0.62 \mathrm{mGy}$ and $0.45 \mathrm{mGy}$, respectively. Moreover, thermal and fast neutrons, which were suggested to have a substantial amount of importance in $\mathrm{PD}$, were identified in irradiation with 10 and $18 \mathrm{MV}$ in the study.[17] These results are compatible with our findings.

\section{Conclusion}

Cervical cancer is the second most common cancer in women after breast cancer based on studies conducted in different countries.[18] Since the incidence of cervical cancer has been increasing among young patients nowadays, PD should be considered to reduce the risk of secondary cancer, especially in younger patients who will be treated with radiotherapy. Particularly, sensitive organs, such as the breast tissue, may be affected by these radiation doses. Therefore, these risks should be evaluated while selecting treatment techniques and parameters, especially MU values. Because the PD could not yet be calculated accurately by TPS, these doses should be measured and recorded.

As a result, our findings suggest that VMAT or 3DCRT techniques are superior to the IMRT technique concerning lower breast PD, especially in younger patients with cervical cancer.

Peer-review: Externally peer-reviewed.

Conflict of Interest: The authors declare that they have no conflict of interest.

Ethics Committee Approval: This study was conducted in accordance with local ethical rules.

Financial Support: This work has no funding source.

Authorship contributions: Concept - Y.S., U.A., H.B.B.; Design - Y.S., M.T.; Supervision - Y.S., H.B.B.; Funding None; Materials - Y.S.; Data collection and/or processing Y.S.; Data analysis and/or interpretation - Y.S., H.B.B., C.K.; Literature search - Y.S., M.T.; Writing - Y.S., M.T.; Critical review - Y.S., H.B.B.

\section{References}

1. WHO. Cancer fact sheets: Cervical cancer: Estimated incidence, mortality and prevalence (5 years) worldwide in 2012. International Agency for Research on
Cancer and World Health Organization; 2012. Available at: http://gco.iarc.fr/today/data/pdf/fact-sheets/ cancers/cancer-fact-sheets-16.pdf

2. Parkin DM, Bray F, Ferlay J, Pisani P. Global cancer statistics, 2002. CA Cancer J Clin 2005:55(2):74-108.

3. Kamangar F, Dores GM, Anderson WF. Patterns of cancer incidence, mortality, and prevalence across five continents: defining priorities to reduce cancer disparities in different geographic regions of the world. J Clin Oncol 2006;24(14):2137-50.

4. Khan FM. The Physics of Radiation Therapy, 3rd edition. Philadelphia; Lippincott Williams \& Wilkins, 2003.

5. Taylor ML, KronT. Consideration of the radiation dose delivered away from the treatment field to patients in radiotherapy. J Med Phys 2011;36(2):59-71.

6. Cho S, Kim SH, Kim CH, Park JG, Park JG, et al. Secondary Cancer Risks in Out-of-field Organs for 3-D Conformal Radiation Therapy. Progress in NUCLEAR SCIENCE and TECHNOLOGY 2011;1:521-4.

7. Stovall M, Blackwell CR, Cundiff J, Novack DH, Palta JR, Wagner LK, et al. Fetal dose from radiotherapy with photon beams: report of AAPM Radiation Therapy Committee Task Group No. 36. Med Phys 1995;22(1):63-82.

8. Jia MX, Zhang X, Yin C, Feng G, Li N, Gao S, et al. Peripheral dose measurements in cervical cancer radiotherapy: a comparison of volumetric modulated arc therapy and step-and-shoot IMRT techniques. Radiat Oncol 2014;9:61.

9. Mansur DB, Klein EE, Maserang BP. Measured peripheral dose in pediatric radiation therapy: a comparison of intensity-modulated and conformal techniques. Radiother Oncol 2007;82(2):179-84.

10. Ceylan C, Güden M, Baş Ayata H, Küçük N, Kiliç A, Engin K. Comparison of different planning techniques and out-of-field doses in bilateral lung irradiation for Wilms' tumor metastatic to the lung. Turkish J Oncology 2012;27(4):202-11.

11. Martín Rincón C, Jerez Sainz I, Modolell Farré I, España López ML, López Franco P, Muñiz JL, et al. Evaluation of the peripheral dose to uterus in breast carcinoma radiotherapy. Radiat Prot Dosimetry 2002;101(1-4):469-71.

12. Lee B, Lee S, Sung J, Yoon M. Radiotherapy-induced secondary cancer risk for breast cancer: 3D conformal therapy versus IMRT versus VMAT. J Radiol Prot 2014;34(2):325-31.

13. Kase KR, Svensson GK, Wolbarst AB, Marks MA. Measurements of dose from secondary radiation outside a treatment field. Int J Radiat Oncol Biol Phys 1983;9(8):1177-83.

14. Banaee N, Nedaie DR, Esmati E, Nosrati H, Jamali $\mathrm{M}$. Dose measurement outside of radiotherapy treat- 
ment field (Peripheral dose) using thermoluminescent dosimeters. Int J Radiat Res 2014;12(4):355-9.

15. Yousif Abdallah YM, Gar-elnabi ME, Bakary AHA, Eltoum AMH, Ali AKM. Calculation of organs radiation dose in cervical carcinoma external irradiation beam using day's methods. GARJMMS 2014;3(5):090-4.

16. Kinhikar R, Gamre P, Tambe C, Kadam S, Biju G, Suryaprakash, et al. Peripheral dose measurements with diode and thermoluminescence dosimeters for intensity modulated radiotherapy delivered with con- ventional and un-conventional linear accelerator. J Med Phys 2013;38(1):4-8.

17. D’Agostino E, Bogaerts R, Defraene G, de Freitas Nascimento L, Van den Heuvel F, Verellen D, et al. Peripheral doses in radiotherapy: A comparison between IMRT, VMAT and Tomotherapy. Radiation Measurements 2013;57:62-7.

18. World Health Organization. Comprehensive cervical cancer control: a guide to essential practice. $2^{\text {nd }}$ edition. WHO; Geneva, 2006. 